\title{
SIRETEA: Una ventana a los métodos de enseñanza y técnicas de evaluación
}

\author{
SIRETEA: A window to teaching methods and assessment techniques \\ María A. Alonso-Lavernia ${ }^{a}$, Yira Muñoz-Sánchez ${ }^{b}$, Iliana Castillo-Pérez ${ }^{c}$, Verónica Martínez- \\ Lazcano $^{d}$
}

\begin{abstract}
:
Currently, teachers and students are faced with changes in education that require the constant development of their skills, since the new educational trends are focused on the development of skills and the evaluation of results, so traditional teaching strategies no longer they are enough to cover the current needs for the formation of the new generations. In this context, it is essential to develop resources that support teaching work in their usual practice and under this premise, a website called SIRETEA has been developed, which provides a theoretical, methodological and practical support on teaching methods, techniques of performance evaluation and observation instruments. In this work, the informative part of the portal is presented with the peculiarity that it has been treated mostly with digital resources in the form of Learning Objects, which offers possibilities for interaction and feedback to the user, being these fundamental characteristics in any process of training.
\end{abstract}

\section{Keywords:}

Teaching methods, Techniques of performance evaluation, Observation instruments

\section{Resumen:}

Actualmente, docentes y alumnos se enfrentan con cambios en la educación que requieren del constante desarrollo de sus habilidades, ya que las nuevas tendencias educativas se enfocan al desarrollo de competencias y a la valoración de resultados, por lo que las estrategias didácticas tradicionales ya no son suficientes para cubrir las necesidades actuales para la formación de las nuevas generaciones. En este contexto, se hace imprescindible el desarrollo de recursos que apoyen la labor docente en su práctica habitual y bajo esta premisa, se ha desarrollado un Portal Web denominado SIRETEA, que brinda un sustento teórico, metodológico y práctico sobre los métodos de enseñanza, técnicas de evaluación del desempeño e instrumentos de evaluación. En este contexto, se hace imprescindible el desarrollo de recursos que apoyen la labor docente en su práctica habitual y bajo esta premisa, se ha desarrollado un Portal Web denominado SIRETEA, que brinda un sustento teórico, metodológico y práctico sobre los métodos de enseñanza, técnicas de evaluación del desempeño e instrumentos de evaluación.

\section{Palabras Clave:}

Métodos de enseñanza, Técnicas de evaluación del desempeño, Instrumentos de evaluación

\footnotetext{
a Autor de Correspondencia, Universidad Autónoma del Estado de Hidalgo, Instituto de Ciencias Básicas e Ingeniería, ORCID: 0000-00029839-8250, Email: marial@uaeh.edu.mx

b Universidad Autónoma del Estado de Hidalgo, Escuela Superior de Ciudad Sahagún, ORCID: 0000-0002-4976-2747, Email: yira@uaeh.edu.mx

c Universidad Autónoma del Estado de Hidalgo, Instituto de Ciencias Básicas e Ingeniería, ORCID: 0000-0002-8130-9231, Email: ilianac@uaeh.edu.mx

d Universidad Autónoma del Estado de Hidalgo, Instituto de Ciencias Básicas e Ingeniería, Email: vlazcano@uaeh.edu.mx
} 


\section{Introducción}

Actualmente, la sociedad es más consciente de la importancia de alcanzar estándares de calidad y aprovechamiento de recursos para mejorar su nivel de competencia, de ahí que los estudiantes del siglo XXI se enfrentan con una educación más desafiante que requiere del constante desarrollo y explotación de sus habilidades. En este nuevo enfoque formativo, el objetivo fundamental es que los alumnos integren las habilidades, destrezas y conocimientos adquiridos en la formación escolar y los apliquen de manera práctica en diferentes escenarios áulicos y no áulicos.

Con los cambios de la cultura escolar, la evaluación se ha convertido en uno de los temas más importantes en el ámbito educativo, ya que bajo este nuevo enfoque se debe valorar la manera en que los estudiantes resuelven situaciones $y$, además, obtener evidencias de los resultados del aprendizaje.1,2

Ante esta situación, algunos críticos argumentan que la aplicación de exámenes clásicos de respuesta fija ya no es una alternativa eficaz para la evaluación, ya que con este tipo de pruebas los estudiantes no demuestran la habilidad del uso del conocimiento que debieran tener.

Debido a lo anterior, es inapelable que las prácticas habituales de evaluación deben cambiar y hacer uso de procedimientos que se puedan incorporar en las actividades docentes diarias y que permitan valorar objetivamente la forma en que los alumnos integran e interpretan el conocimiento. Por lo tanto, para la evaluación del aprendizaje se requieren técnicas que faciliten al profesor la valoración directa del trabajo de los alumnos mediante la observación de evidencias, siendo en este contexto donde las Técnicas de Evaluación Alternativas (TEA) encajan con este nuevo modelo. 3,4,5

Las técnicas de evaluación alternativas son métodos usados en el proceso educativo e integrados a las actividades diarias de los alumnos, que permiten valorar el conocimiento y desarrollo de habilidades. Con respecto a estas técnicas existen dos alternativas, las técnicas para la evaluación del desempeño y las técnicas de observación, las últimas sirven como complemento para las primeras, por lo que debe establecerse una correcta relación entre ambas. La pertinencia del uso de estas técnicas se define en función de la finalidad que se persigue y de acuerdo con los aspectos que se desee evaluar. 6

A través de las técnicas de evaluación del desempeño, los profesores obtienen evidencias mediante productos elaborados por los alumnos. Con estos productos los estudiantes demuestran los saberes que poseen. Así mismo, el profesor utiliza técnicas de observación para valorar el aprendizaje, enfatizar las fortalezas, así como evaluar la capacidad, la experiencia y el nivel de conocimiento de cada uno de los estudiantes. Con las técnicas de observación se hace uso de instrumentos que sirven para establecer los aspectos a valorar en las técnicas de desempeño.

Considerando la relevancia que tiene la educación para el desarrollo de la sociedad, ha sido un esfuerzo permanente el brindar propuestas para mejorar o facilitar los procesos de formación en general y en particular, aquellos dedicados a las estrategias evaluativas, pues son éstas precisamente, las que utilizará el profesor para valorar el aprendizaje de los estudiantes.

Las alternativas presentadas en los últimos años para difundir y aplicar las estrategias evaluativas se han materializado fundamentalmente en el desarrollo de portales web; sitios web o páginas web, todas ellas en correspondencia con las posibilidades tecnológicas actuales y los propósitos del trabajo, sin embargo, esta opción no deja atrás las propuestas también impactantes a través de artículos 0 múltiples documentos en la Web. 7-10, 11-13, 14-16, 17-19

En cuanto al enfoque de las propuestas, también son muy versátiles debido a que su propósito puede ser informativo, de interacción o mixta, donde los informativos tienen el fin de brindar un apoyo a la formación docente en cuanto al conocimiento de estas estrategias evaluativas mientras que las interactivas buscan el desarrollo de habilidades en la creación de instrumentos de evaluación y finalmente, las mixtas reúnen las ventajas de los tipos anteriores. 7-9, 11,12, $15-18$

De acuerdo con el tema que tratan, la mayoría presentan aspectos relacionados con las técnicas y/o instrumentos de evaluación, sin embargo, a pesar de que estos aspectos son muy abordados hoy en día en la literatura, la forma de presentar la información es mayoritariamente textual. 9, 11-18

Este último aspecto es atendido en el presente trabajo, pues se brinda información acerca de algunas estrategias didácticas a través de recursos didácticos digitales en forma de Objetos de Aprendizaje (OA), mismo que incluyen además del contenido esperado, otros medios de formación como son actividades y evaluaciones, características que promueven la interacción con el usuario y sirven para retroalimentar su desempeño. 


\section{Proyecto SIRETEA}

El Portal de Recomendación de Técnicas de Evaluación Alternativas (SIRETEA) es un proyecto que se encuentra en desarrollo actualmente y que pretende dar respuesta a las necesidades de uso de las estrategias evaluativas por parte de los docentes en el proceso de formación de los estudiantes. Su propósito es el de servir a los docentes como herramienta teórica, metodológica y práctica para apoyarlos en la correcta selección de técnicas de evaluación alternativas, mediante sugerencias automáticas basadas en la relación entre métodos de enseñanza, técnicas de evaluación del desempeño e instrumentos de evaluación. Así mismo, se incluyen plantillas para facilitar la creación de ejemplos de instrumentos y permitir llevar a cabo tareas de gestión en línea de estos. Este portal se encuentra publicado en http://cidecame.uaeh.edu.mx/Siretea/Principal.html.

Un aspecto que destacar en este proyecto es que se puedan sugerir recomendaciones de las técnicas de evaluación alternativas basándose en su relación con los métodos de enseñanza que utiliza el docente para la transmisión de los saberes, haciendo de esta manera, que las técnicas para la observación y valoración de resultados se correspondan con las actividades de enseñanza, estableciendo así la integración de actividades, técnicas e instrumentos.

SIRETEA procura ser una herramienta que permita el desarrollo de habilidades en el uso y aplicación de estas técnicas en la práctica docente, de la cual puedan asirse los docentes para enfrentar el cambio actual de la cultura escolar.

Su arquitectura computacional consta de dos secciones:

1. Sección pública del sistema, en la cual el usuario puede realizar el registro de usuario, el inicio de sesión, acceder a la información teórica de consulta y a los ejemplos de estrategias evaluativas e instrumentos de evaluación que los usuarios registrados compartan como públicos.

2. Sección privada del sistema, para hacer uso de ella el usuario deberá previamente registrarse y acceder con su dirección de correo electrónico y contraseña. Dependiendo del rol o tipo de usuario se pueden realizar tareas como crear y gestionar estrategias evaluativas e instrumentos de evaluación.

Este portal pretende servir como referente de las técnicas de evaluación alternativas y su relación con los métodos de enseñanza, integrando los siguientes atributos:
- Teórico
- Metodológico
- De gestión

Esta arquitectura puede ser visualizada por el usuario a través de interfaces gráficas y formularios, mismos que se muestra en la Figura 1.

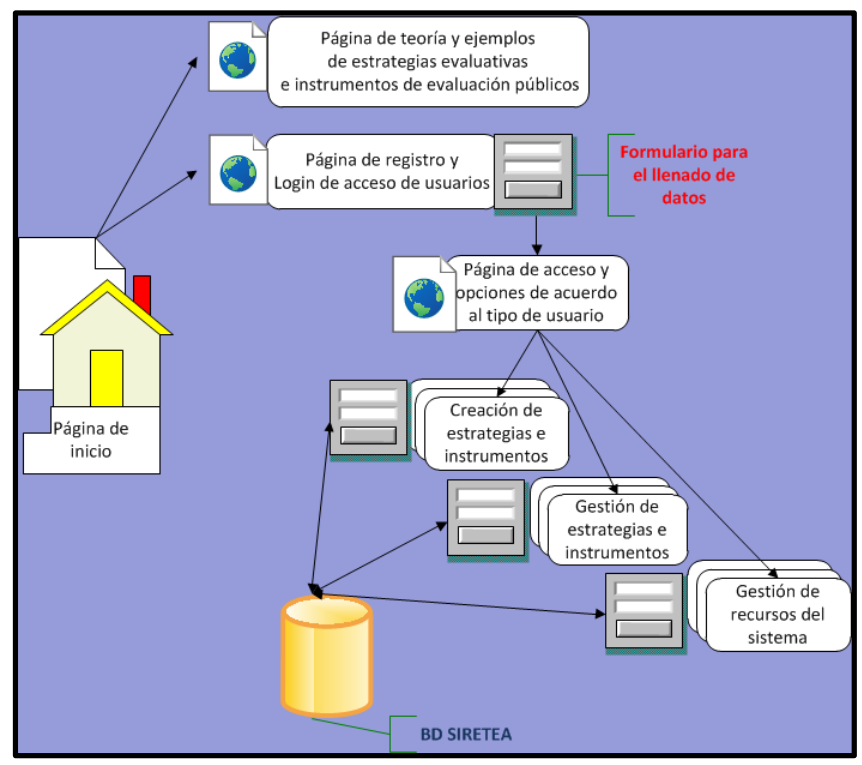

Figura 1. Principales interfaces gráficas y formularios Web que componen el SIRETEA

En cuanto a los atributos teóricos, este sistema ofrece actualmente, información de consulta para que los docentes cuenten con un marco teórico enfocado principalmente a las técnicas de evaluación alternativas y como complemento se ofrece la definición conceptual de algunos métodos de enseñanza. De manera específica, se abordan:

1. Métodos de enseñanza:

- Lección magistral

- Resolución de problemas

- Aprendizaje orientado a proyectos

- Estudio basado en casos

2. Técnicas de evaluación del desempeño:

- Cuadro sinóptico

- Debate

- Diario

- Ensayo

- Mapa conceptual

- Mapa mental

- Portafolio

- Proyecto

- Resumen

- Texto paralelo 
- Técnica de la pregunta

- Técnica expositiva

\section{Técnicas de observación:}

- Escala de rango

- Lista de cotejo

- Rúbrica

Con respecto a los atributos metodológicos, en este portal se podrán proporcionar recomendaciones automáticas para el uso de TEA, las cuales están basadas en la previa elección de un método de enseñanza y se fundamentan en las relaciones existentes entre los métodos, las técnicas de evaluación del desempeño y los instrumentos de evaluación. De esta manera, se ofrece un referente metodológico que apoye a los profesores en la selección de TEA acordes a las actividades que desarrollarán con sus alumnos dentro o fuera del escenario áulico.

Este portal permitirá la creación de instrumentos de evaluación a partir de plantillas, en las que se muestra la estructura general del instrumento y se propone contenido para establecer los aspectos y escalas de valoración, además es posible que el usuario modifique y adecúe este contenido de acuerdo con sus necesidades.

Además de permitir la creación de instrumentos, se tendrán otros atributos de gestión que permiten el manejo de las plantillas, brindando operaciones tales como:

$$
\begin{aligned}
& \text { - Guardar } \\
& \text { - } \text { Buscar } \\
& \text { - } \text { Modificar } \\
& \text { - Exportar } \\
& \text { - Eliminar }
\end{aligned}
$$

Las técnicas y herramientas recomendadas, así como las plantillas personalizadas por los usuarios serán respaldadas en una base de datos que protege $y$ asegura la información, facilitando la generación de estadísticas de uso del sistema.

Los atributos teóricos, metodológicos y de gestión, convierten al portal en una herramienta integradora, accesible y proactiva que puede ser utilizada en el trabajo docente.

En este trabajo, se presenta la sección informativa del Portal SIRETEA, la cual corresponde con la sección pública.

\section{Recursos en SIRETEA}

Los recursos didácticos que se ofrecen en el Portal SIRETEA son Objetos de Aprendizaje (OA), principalmente. A través de estos $O A$ se abordan diferentes Métodos de enseñanza, Técnicas de evaluación del desempeño y Técnicas de observación.

Los $\mathrm{OA}$, a diferencia de otro tipo de materiales, incluyen elementos que permiten a quien los utiliza reafirmar $y$ retroalimentar lo aprendido en dicho OA.

A continuación, se explican los elementos que integran los OA desarrollados y la metodología bajo la cual fueron creados.

\section{Estructura de los Objetos de Aprendizaje}

Cada uno de los OA tiene una estructura definida, la cual consta de los siguientes elementos:

- Portada: es la presentación de OA y muestra la institución que lo desarrolló, así como el tema que se aborda en el mismo.

- Objetivo de Aprendizaje: define el aprendizaje que logrará el usuario con el uso del OA.

- Contenido: incluye la organización y presentación del tema a través textos y multimedios, de acuerdo con el objetivo de aprendizaje.

- Actividades: comprenden un grupo de dinámicas a realizar fuera del contexto del $O A$ que permitirá al usuario reforzar lo visto en el contenido y representan un medio más para alcanzar el objetivo de aprendizaje.

- Evaluación: incluye diferentes ejercicios para comprobar si se alcanzó el objetivo de aprendizaje del objeto.

- Glosario: describe las palabras técnicas o poco comunes utilizadas en el contenido del OA para su mejor comprensión.

- Referencias: incluye la bibliografía utilizada en el desarrollo del contenido y cualquier otro medio que amplíe conceptos 0 criterios presentados en el OA.

- Créditos: muestra los nombres de los autores y evaluadores del $\mathrm{OA}$.

Cada uno de los elementos descritos tributa al conocimiento, habilidad $y / 0$ aptitud que se pretende alcanzar con el uso del OA y que se define en el objetivo de aprendizaje. 20 


\section{Metodología para el Desarrollo de Objetos de Aprendizaje (MEDOA)}

Los OA se desarrollaron bajo la metodología MEDOA, la cual se basa en un modelo mixto que combina las arquitecturas de cascada y espiral, como se muestra en la Figura 2.

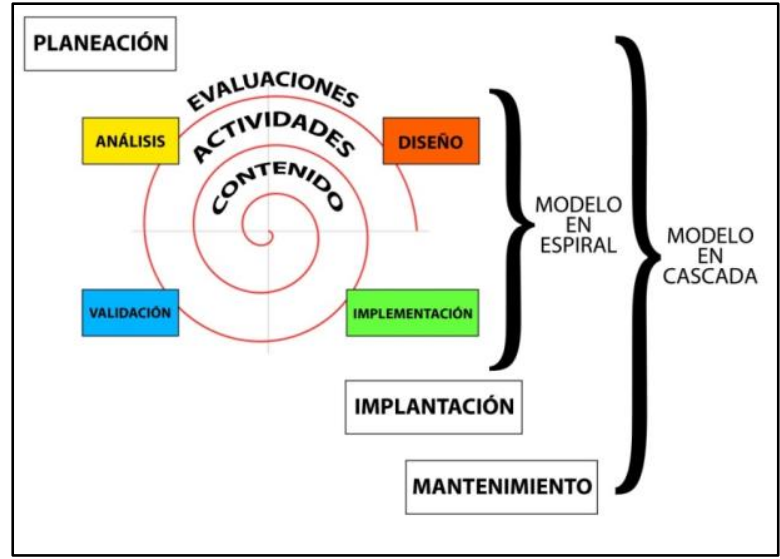

Figura 2. Arquitectura mixta de MEDOA

Tal cual se puede apreciar en la Figura 2, cada OA desarrollado en SIRETEA inicia con la fase de Planeación y de modo secuencial se continúa con la ejecución de cuatro fases que integran el modelo en espiral y que son: Análisis, Diseño, Implementación y Validación. Estas cuatro fases se llevan a cabo de forma iterativa, para el contenido, las actividades y las evaluaciones es decir, se realizan las cuatro fases (Análisis, Diseño, Implementación y Validación) para el desarrollo del contenido, posteriormente y con base a lo abordado en el contenido se transita por las mismas etapas para la creación de actividades y después para las evaluaciones. Finalmente, se continúa con las fases de Implantación y Mantenimiento, propias del modelo en cascada. 21

Todas las fases MEDOA integran el ciclo de vida del software educativo y representan todo el proceso desde el inicio donde se proyecta todo el desarrollo hasta la puesta en marcha para su uso educativo. Cada fase está compuesta por un conjunto de actividades, denominadas en la metodología MEDOA como Pasos, las cuales facilitan la obtención de un subproducto necesario dentro del desarrollo de los OA.

\section{Objetos de Aprendizaje}

En este apartado, se darán a conocer los OA publicados en los tres temas que se abordan en el portal, a través de una breve descripción de la importancia de la técnica o método que se expone.

\section{Métodos de Enseñanza}

Dada la relevancia actual de fomentar el aprendizaje significativo durante los procesos de enseñanza y aprendizaje, se desarrollaron los siguientes objetos: Resolución de Problemas y Estudio basado en Casos.

La resolución de problemas permite fomentar en los estudiantes las capacidades de aprender a aprender, por lo que en el OA construido, cuya portada se muestra en la Figura 3, se describe de manera detallada esta técnica para aprender a utilizarla.

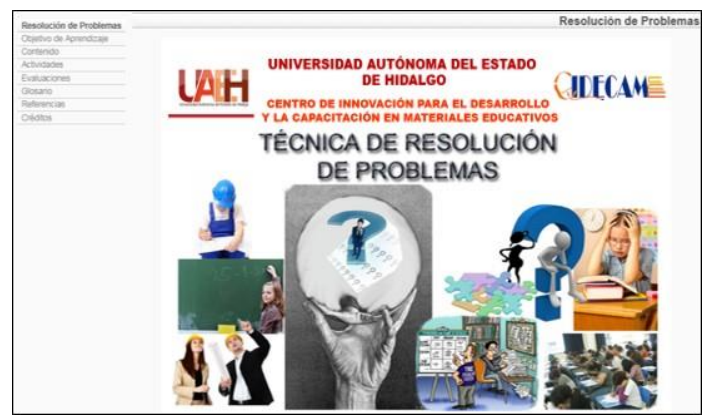

Figura 3. Portada de OA de la Técnica de Resolución de Problemas

A través de la resolución de problemas, se enfrenta al estudiante a una situación específica en la que se plantea un problema para que lo comprenda, lo valore y lo resuelva. EI OA desarrollado para esta técnica y cuya portada se muestra en la Figura 4, permite comprender en qué consiste la misma, su uso y su aplicación.

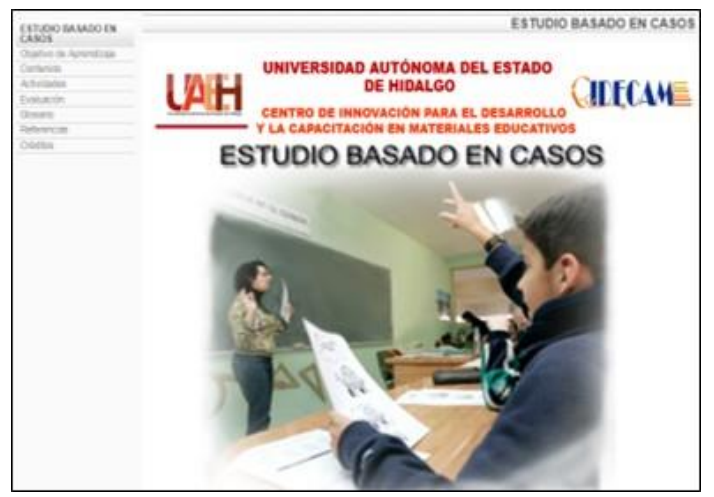

Figura 4. Portada de OA de Estudio basado en Casos

\section{Técnicas de Evaluación del Desempeño}

Las técnicas de evaluación del desempeño valoran el conocimiento y habilidades adquiridos por los estudiantes en el proceso de enseñanza-aprendizaje a través de la elaboración de una tarea, producto, respuesta o actuación. Con el desarrollo o creación de 
los mismos, el alumno integra conocimientos, destrezas, actitudes, valores y habilidades, además demuestra lo que es capaz de hacer y el nivel de logro del aprendizaje que posee. Para este rubro, se desarrollaron los siguientes OA: Cuadro sinóptico, Debate, Diario, Ensayo, Mapa conceptual, Mapa mental, Portafolio, Proyecto, Resumen, Texto paralelo, Técnica de la pregunta, Técnica expositiva. 22

El cuadro sinóptico es una técnica o herramienta de aprendizaje en forma gráfica de un texto, los cuales permiten visualizar las ideas principales e importantes de un texto. El OA desarrollado, cuya portada se muestra en la Figura 5, indica cómo realizar correctamente un cuadro sinóptico, qué características lo identifican, su estructura y tipos.

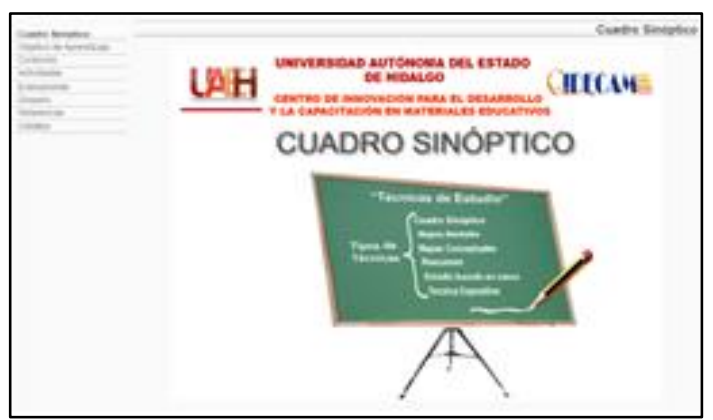

Figura 5. Portada de OA del Cuadro Sinóptico

El debate es un acto de comunicación que consiste en la discusión de un tema polémico entre dos o más personas con carácter argumentativo, utilizado para conocer las distintas posturas. EI OA sobre este tema, cuya portada se muestra en la Figura 6 , describe cómo utilizar esta técnica.

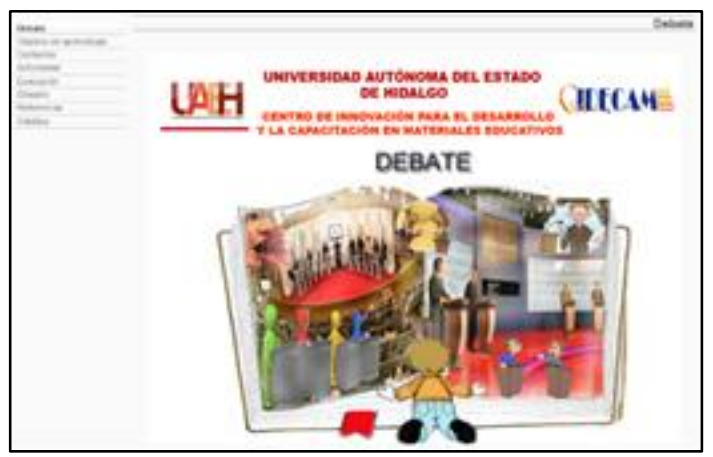

Figura 6. Portada de OA de Debate

El diario es utilizado en el proceso educativo para que el estudiante exprese sus ideas y dudas sobre los temas vistos en clase y el docente pueda adaptarse a todo tipo de circunstancias, elevando así el nivel de aprendizaje de sus estudiantes. La Figura muestra la portada del OA sobre la técnica didáctica del Diario.

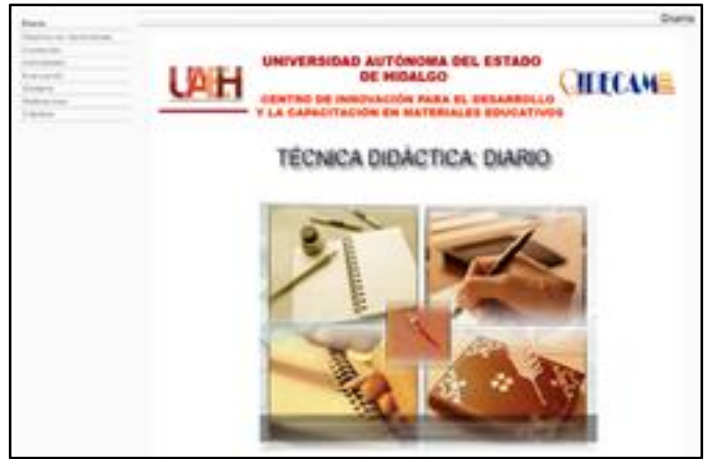

Figura 7. Portada del OA de la Técnica del Diario

La importancia de aprender a realizar un ensayo es adquirir y desarrollar las competencias textual, semántica y argumentativa para superar el déficit teórico y técnico que presentan los estudiantes en la producción y comprensión de textos, así como en la argumentación del pensamiento propio. El OA desarrollado, cuya portada se muestra en la Figura 8 , permitirá al usuario comprender en qué consiste la técnica, su uso y su aplicación.

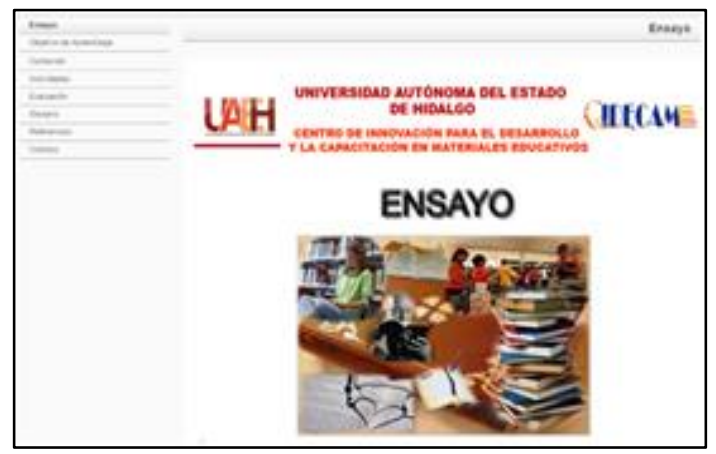

Figura 8. Portada del OA de la Técnica del Ensayo

La Figura muestra la portada del OA del mapa conceptual. Dicha técnica de estudio se utiliza en diferentes niveles educativos ya que permite sintetizar, organizar y estructurar de manera gráfica las relaciones significativas entre los conceptos, permitiendo interrelacionar y fijar el conocimiento del contenido estudiado.

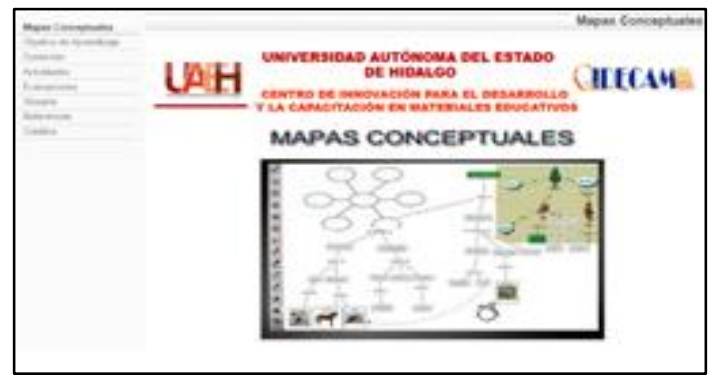

Figura 9. Portada del OA de Mapas Conceptuales

El mapa mental es un instrumento que permite aprender de una manera clara, amena e ilustrativa, ya que brinda 
información global de un tema. Esta metodología ofrece una secuencia didáctica estructurada que facilita el proceso de enseñanza-aprendizaje, ayuda a enfocar las necesidades que tiene el que aprende creando interés y satisfacción al mismo tiempo. El OA desarrollado, cuya portada se muestra en la Figura 10, incluye la descripción detallada para su uso.

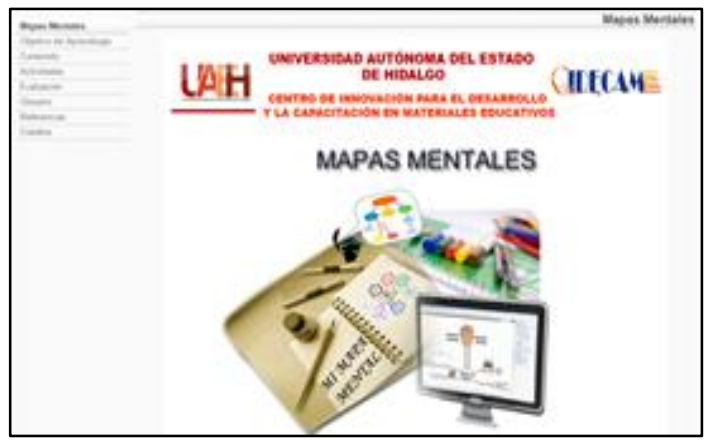

Figura 10. Portada del OA de Mapas Mentales

Un proyecto es un instrumento útil para evaluar el aprendizaje de los alumnos, toda vez que permite verificar las capacidades de representar objetivos a alcanzar, caracterizar propiedades de lo que será trabajado, anticipar resultados intermedio y finales, escoger estrategias adecuadas para solucionar la problemática, ejecutar acciones para alcanzar resultados específicos y evaluar condiciones para la solución del problema. La Figura muestra la portada del OA donde se describe a detalle esta técnica.

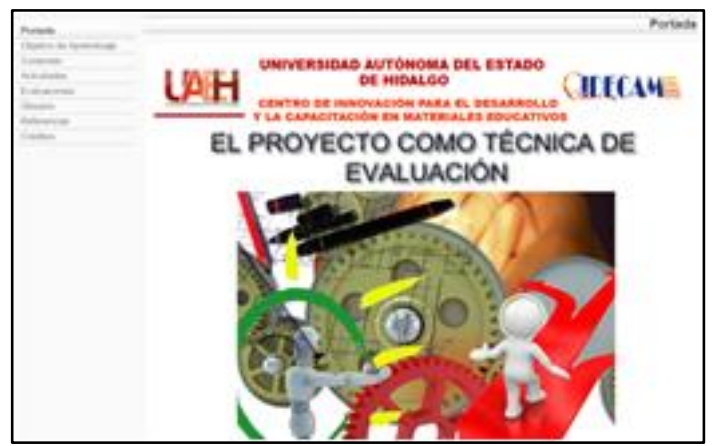

Figura 11. Portada del OA de la Técnica de Proyecto

La técnica del resumen genera una exposición abreviada, precisa y ordenada de un texto considerando sólo las ideas más importantes y necesarias. Con esta técnica el estudiante pasa de ser un mero receptor pasivo a ser un procesador de información que valora, critica, que amplía, compara y reconstruye la información. El OA sobre este tema, cuya portada se muestra en la Figura 12, describe cómo utilizar esta técnica.

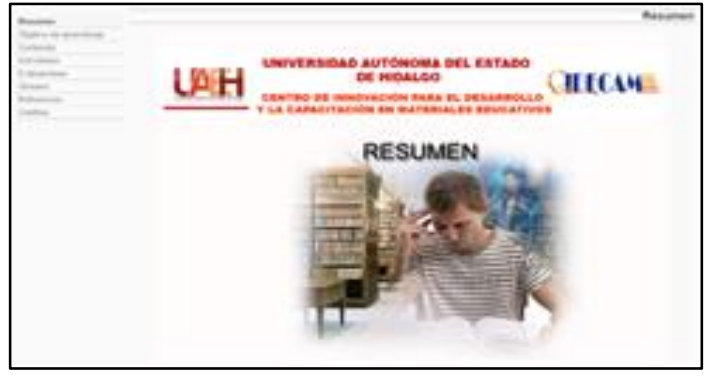

Figura 12. Portada del OA de la Técnica del Resumen

La construcción de textos paralelos es muy importante ya que con esta se propicia la investigación documental, con su realización se construyen conocimientos, también propicia la apropiación del proceso de aprendizaje dando sentido a lo que se aprende y la expresión de la experiencia educativa a través de un producto propio elaborado con flexibilidad y creatividad. La Figura contiene la portada del OA de esta técnica.

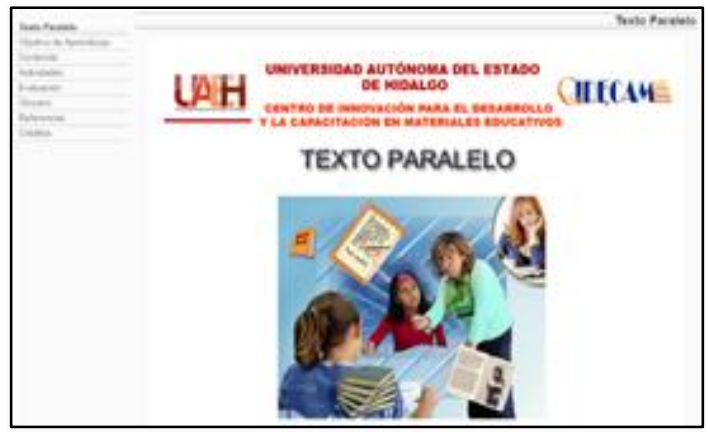

Figura 13. Portada del OA de Texto Paralelo

La Figura presenta la portada del OA de la técnica de la pregunta, la cual se considera un instrumento para evaluar las habilidades metacognitivas de una forma clara y directa. Esta técnica permite comprobar el nivel alcanzado en la competencia que se desea desarrollar en el alumno, de tal forma, que el docente puede decidir si efectúa una retroalimentación o no, con el fin de alcanzar los objetivos planteados.

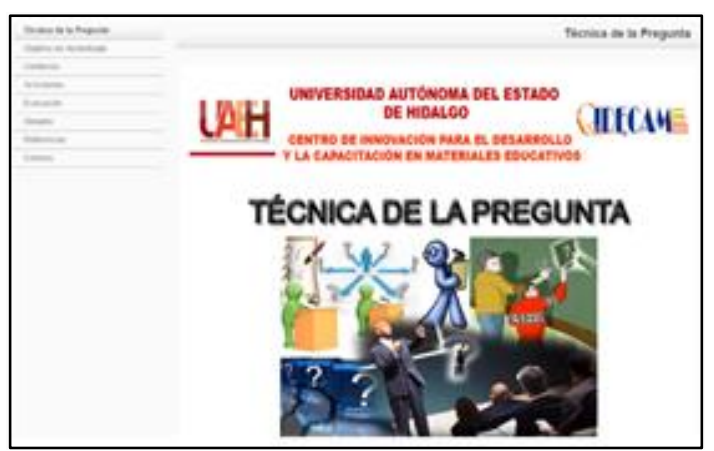

Figura 14. Portada del OA de la Técnica de la Pregunta

De entre las técnicas tradicionales de enseñanza, una de las más utilizadas es la denominada expositiva. Esta técnica es especialmente apropiada cuando se desea 
una comunicación directa entre un expositor y un público que recibe información sobre un tema específico previamente preparado. EI OA desarrollado, cuya portada se muestra en la Figura 15, permitirá al usuario comprender en qué consiste la técnica, su uso y su aplicación.

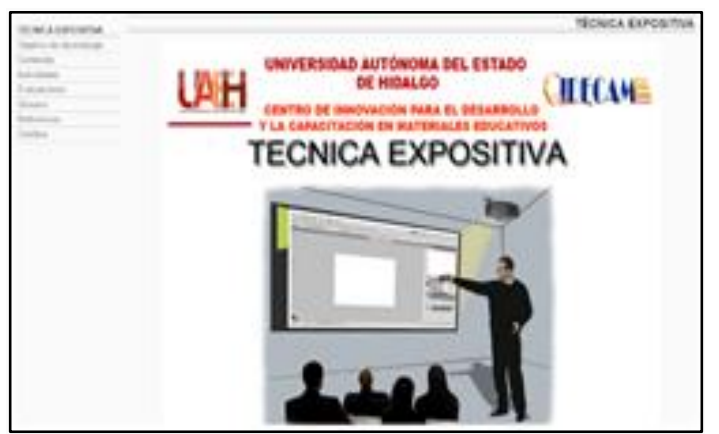

Figura 15. Portada del OA de la Técnica Expositiva

\section{Instrumentos de evaluación}

Existen diversas técnicas para observar dentro del proceso educativo, siendo las más comunes: Lista de Cotejo, Rúbrica y la Escala de Rango, las cuales tienen como objetivo común, establecer los criterios para evaluar conocimientos, habilidades y actitudes de los estudiantes. Por tanto, estas técnicas son un complemento de las técnicas de evaluación del desempeño.

La escala de rango es un instrumento de observación que consiste en juzgar una serie de ítems o características que han sido cuidadosamente seleccionadas, de manera que forme un juicio válido y fiable que permita medir el nivel de aprendizaje del alumno, ya sea a través de comportamientos, habilidades y/o actitudes. La Figura muestra la portada del OA donde se describe a detalle el uso de este instrumento de evaluación.

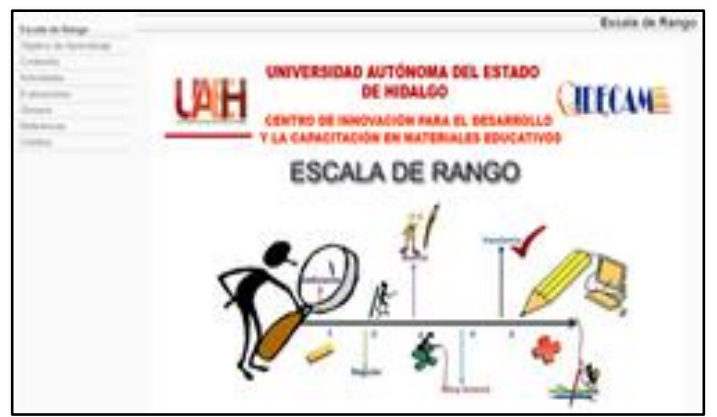

Figura 16. Portada del OA de la Escala de Rango

La lista de cotejo es un instrumento estructurado que registra la ausencia o presencia de un determinado rasgo, conducta o secuencia de acciones, es apropiado para registrar desempeños de acciones corporales, destrezas motoras, o bien los resultados o productos de trabajos realizados. EI OA sobre este tema, cuya portada se muestra en la Figura 17, describe cómo utilizar crear y utilizar este instrumento.

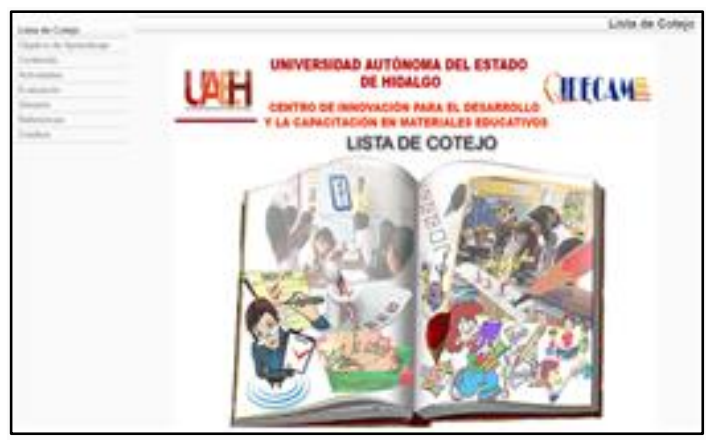

Figura 17. Portada del OA de Lista de Cotejo

La rúbrica es una matriz de valoración que consiste en un listado de criterios específicos que permiten valorar el aprendizaje, el desarrollo de competencias y capacidades logradas por el estudiante. EI OA desarrollado sobre este instrumento, cuya portada se presenta en la Figura 18, permitirá al usuario comprender sus elementos, cómo construirla y cómo aplicarla.

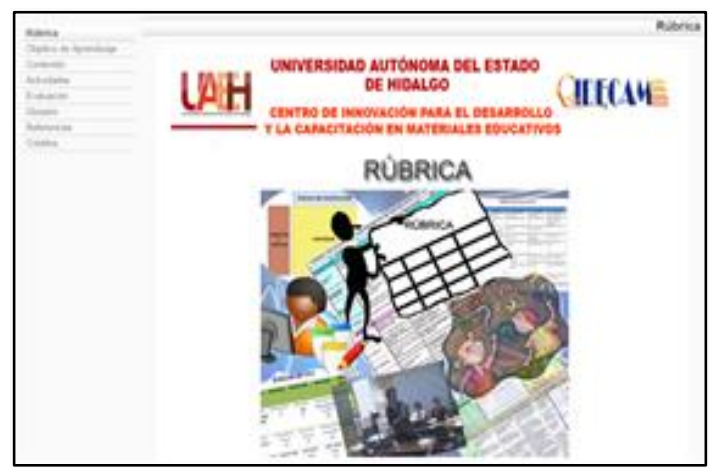

Figura 18. Portada del OA de la Rúbrica

Los recursos para los métodos de enseñanza de Lección Magistral y de Aprendizaje Orientado a Proyectos, así como la técnica de evaluación del desempeño del Portafolio se encuentran en formato PDF.

\section{Conclusiones}

La formación de docentes en estrategias didácticas que mejoren su actividad profesional hoy en día es preponderante, pues así lo demandan los modelos educativos modernos. De ahí, la relevancia que puedan tener las aportaciones que se realicen en este sentido. Sin embargo, buscar soluciones más integradoras, accesible y proactiva para el docente facilitará su formación y, en consecuencia, permitirá alcanzar la 
calidad que requieren los procesos de enseñanza y aprendizaje.

Aunque en este trabajo, se presenta sólo el portal informativo, los Objetos de Aprendizaje utilizados para ello favorece la formación en algunos métodos de enseñanza, técnicas e instrumentos de evaluación.

Este portal es utilizado en la formación de los estudiantes de la Maestría en Tecnologías de la Información para la Educación y está disponible para todo público interesado en estos temas.

\section{Trabajos Futuros}

Como consecuencia al auge y al impacto que tienen las tecnologías digitales en beneficio de la educación, se abren diversas líneas para que el portal SIRETEA continúe favoreciendo el aprendizaje respecto a Métodos de enseñanza, Técnicas de evaluación del desempeño y Técnicas de observación a través de las tecnologías actuales y futuras, con base a las necesidades de conocimiento que van surgiendo.

Una primera línea para darle continuidad a este proyecto es el desarrollo de OA para los tres recursos que aún se encuentran en formato PDF; específicamente, la Lección Magistral, el Aprendizaje Orientado a Proyectos y el Portafolio. Además, incluir otros métodos de enseñanza, técnicas e instrumentos de evaluación.

Continuar con los trabajos previstos en el Proyecto SIRETEA en cuanto al ambiente para el manejo de Instrumentos de Observación y la posibilidad de recomendar estos últimos en correspondencia con el método de enseñanza y la técnica de evaluación utilizada.

\section{Referencias}

[1] M. Á. Santos, La evaluación como aprendizaje: Cuando la flecha impacta en la diana, Narcea Ediciones, 2014.

[2] K. E. Gallardo, Evaluación del aprendizaje: retos y mejores prácticas, Editorial Digital del Tecnológico de Monterrey, 2018.

[3] B. S. López y E. M. Hinojosa, Evaluación del aprendizaje: alternativas y nuevos desarrollos, Trillas, 2005.

[4] F. Díaz Barriga y G. Hernández, Estrategias docentes para un aprendizaje significativo : una interpretación constructivista, McGraw-Hill, 2001

[5] J. . L. Pulgar, Evaluación del aprendizaje en educación no formal: Recursos prácticos para el profesorado, Narcea Ediciones, 2005.

[6] Ministerio de Educación de Guatemala y USAID, Herramientas de evaluación en el aula, Ministerio de Educación de Guatemala, 2011.

[7] Teachnology, Inc, «teAchnology: The Online Teacher Resource,» 2019. [En línea]. Available: http://www.teach-nology.com/.

[8] ALTEC, «Rubistar:Create Rubrics for your Project-Based Learning Activities,» 2019. [En línea]. Available: http://rubistar.4teachers.org/index.php.

[9] Evalfor, «evalCOMIX: Servicio Web para la e-evaluación,» 2019. [En línea]. Available: http://evalcomix.uca.es/.

[10] UNESCO, «Evaluaciones del aprendizaje,» Learning Portal, 2019. [En línea]. Available: https://learningportal.iiep.unesco.org/es/fichaspraticas/monitorear-el-aprendizaje/evaluaciones-del-aprendizaje.
[11] EDUCREA, «EDUCREA: Estilos y Metodologías de Aprendizaje,» 2019. [En línea]. Available: https://educrea.cl/estilos-ymetodologias-de-aprendizaje/.

[12] M. L. Argudín, «Habilidades Docentes,» 2019. [En línea]. Available: //hadoc.azc.uam.mx.

[13] Reazon Systems, Inc., «Rcampus,» 2019. [En línea]. Available: https://www.rcampus.com/index.cfm.

[14] A. Y. Moreta, «Técnicas de Evaluación (alternativa, auténtica, constructiva),» 2011. [En línea]. Available: https://innovatedocente.webnode.es/products/tecnicas-de-evaluacionalternativa-autentica-constructiva-/.

[15] EcuRed, «EcuRed: Métodos de enseñanza,» 2019. [En línea] Available:

https://www.ecured.cu/M\%C3\%A9todos_de_ense\%C3\%B1anza.

[16] Grupo Telesup, «Instrumentos y técnicas de evaluación educativa,» 2019. [En línea]. Available:

https://escueladeposgrado.edu.pe/blog/instrumentos-y-tecnicas-deevaluacion-educativa/.

[17] R. García-Perales, «Diseño y construcción de un instrumento de evaluación de la competencia matemática: aplicabilidad práctica de un juicio de expertos,» Scielo, vol. 26, nº 99, 2018.

[18] C. Hamodi, V. . M. López-Pastor y A. T. López-Pastor, «Medios, técnicas e instrumentos de evaluación formativa y compartida del aprendizaje en educación superior. Perfiles educativos, 37(147), 146161. Recuperado en 11 de marzo de 2019, de,» vol. 37, n 149, pp. 146-161, 2015.

[19] P. Araque-Marín, S. A. Torijano-Gutiérrez y N. Arango-Londoño, «Diseño e implementación de rúbricas como instrumento de evaluación del curso de Química General e inorgánica para estudiantes de ingeniería,» EIA, vol. 16, nº 31, pp. 131-143, 2019.

[20] M. d. 1. A. Alonso, I. Castillo, M. J. Pozas, V. Martínez y Y. Muñoz, Objetos de Aprendizaje: Una Guía Práctica para su Desarrollo, Universidad Autónoma del Estado de Hidalgo, 2013.

[21] M. A. Alonso, I. Castillo, V. Martínez y Y. Muñoz, «MEDOA: Metodología para el Desarrollo de Objetos de Aprendizaje” (),,» de 12 da Conferencia Iberoamericana en Sistemas, Cibernética, Florida EUA, 2013.

[22] R. Anijovich, M. Malbergier y C. Sigal, Una Introducción a la Enseñanza para la, Primera ed., Buenos Aires: Argentina: Fondo de Cultura Económica de, 2004. 